\title{
A CONVERSE TO LEBESGUE'S DOMINATED GONVERGENCE THEOREM
}

\author{
DWIGHT B. GOODNER
}

(Received 24 November 1965)

Let $(X, B, m)$ be a measure space and let $f(x)$ be a real-valued or complex-valued measurable function on $X$. A non-negative measurable function $s(x)$ will be said to dominate $f(x)$ provided $|f(x)| \leqq s(x)$ for almost all $x$ in $X$. The function $s(x)$ will be said to dominate the sequence $\left\{f_{n}(x)\right\}_{n \in N}$, $N=\{1,2, \cdots\}$, provided it dominates each $f_{n}(x)$ in the sequence. Unless otherwise specified, each integral will be over $X$ with respect to $m$.

Lebesgue's theorem on dominated convergence [1], a cornerstone of modern analysis, says (cf. [3, p. 29]) that if the sequence $\left\{f_{n}(x)\right\}_{n \in N}$ of realvalued measurable functions on $X$ is dominated by an integrable function, then

and

$$
\int \liminf _{n \rightarrow \infty} f_{n}(x) \leqq \liminf _{n \rightarrow \infty} \int f_{n}(x)
$$

$$
\int \limsup _{n \rightarrow \infty} f_{n}(x) \geqq \limsup _{n \rightarrow \infty} \int f_{n}(x) .
$$

Furthermore, if $\lim _{n \rightarrow \infty} f_{n}(x)$ exists for almost all $x$ in $X$, then

$$
\int \lim _{n \rightarrow \infty} f_{n}(x)=\lim _{n \rightarrow \infty} \int f_{n}(x) \text {. }
$$

The purpose of this note is to prove a converse to Lebesgue's theorem. Our proof is based on the following theorem of $\mathrm{B}$. C. Rennie [2]: If the sequence $\left\{f_{n}(x)\right\}_{n \in N}$ of real-valued or complex-valued integrable functions tends almost everyzehere to a function $f(x)$ on $X$, and if $\lim _{n \rightarrow \infty} \int g(x) f_{n}(x)=\int g(x) f(x)$ for each bounded measurable function $g(x)$ on $X$, then each infinite subsequence of $\left\{f_{n}(x)\right\}_{n \in N}$ contains an infinite sub-subsequence which is dominated by an integrable function.

We note that if the $f_{n}(x)$ 's are real-valued only, then in Rennie's proof it suffices to consider only those real-valued measurable functions $g(x)$ such that $|g(x)|=1$ for each $x$ in $X$.

THEOREM. Let $\left\{f_{n}(x)\right\}_{n \in N}$ be a sequence of extended-real-valued integrable functions on $X$ such that 


$$
r(x)=\limsup _{n \rightarrow \infty} f_{n}(x) \text { and } s(x)=\liminf _{n \rightarrow \infty} f_{n}(x)
$$

are integrable, and let

and

$$
R_{n}(x)=\left(r \cup f_{n}\right)(x)=\max \left\{r(x), f_{n}(x)\right\}
$$

$$
S_{n}(x)=\left(s \cap f_{n}\right)(x)=\min \left\{s(x), f_{n}(x)\right\}
$$

for each $x$ and $n$. If

$$
\lim _{n \rightarrow \infty} \int g(x) R_{n}(x)=\int g(x) r(x) \text { and } \lim _{n \rightarrow \infty} \int g(x) S_{n}(x)=\int g(x) s(x)
$$

for each measurable function $g(x)$ with range $\{1,-1\}$, then each infinite subsequence of $\left\{f_{n}(x)\right\}_{n \in N}$ contains an infinite sub-subsequence which is dominated by an integrable function.

Proof. Let $A$ be the set of all $x$ in $X$ such that $r(x)$ or $s(x)$ or at least one $f_{n}(x)$ is infinite. Since $A$ is of measure zero, we may redefine the functions on $A$ without changing the values of their integrals on $X$ and, hence, without loss of generality in our proof. For each $x$ in $A$ and each $n$ in $N$ let $f_{n}(x)=0$. Then $r(x)$ and $s(x)$ and all $f_{n}(x), R_{n}(x)$ and $S_{n}(x)$ are finite everywhere.

In the sequel $N(1), N(2)$ and $N(3)$ will denote infinite subsets of $N$ such that $N(1) \supset N(2) \supset N(3)$. Let $N(1)$ be any infinite subset of $N$. For each $n \in N(1)$ the function $R_{n}(x)$ is the least upper bound of two integrable functions and is, therefore, integrable. Since $r(x)=\limsup _{n \rightarrow \infty} f_{n}(x)=$ lim $\sup _{n \rightarrow \infty} R_{n}(x)$ and since $R_{n}(x) \geqq r(x)$ for each $x$ in $X$, we see that $\lim _{n \rightarrow \infty} R_{n}(x)=r(x)$. By hypothesis $\lim _{n \rightarrow \infty} \int g(x) R_{n}(x)=\int g(x) r(x)$ for each measurable function $g(x)$ with range $\{1,-1\}$. Thus the sequence $\left\{R_{n}(x)\right\}_{n \in N}$ satisfies the hypotheses of Rennie's theorem for real-valued functions, and it follows that the subsequence $\left\{R_{n}(x)\right\}_{n \in N(1)}$ contains an infinite sub-subsequence $\left\{R_{n}(x)\right\}_{n \in N(2)}$ dominated by an integrable function $R(x)$.

By an argument similar to the one above we can show that $\left\{S_{n}(x)\right\}_{n \in N(2)}$ contains a subsequence $\left\{S_{n}(x)\right\}_{n \in N(3)}$ dominated by an integrable function $S(x)$. It follows that $-S(x) \leqq f_{n}(x) \leqq R(x)$ for all $x$ and all $n \in N(3)$, and, hence, that the sub-subsequence $\left\{f_{n}(x)\right\}_{n \in N(3)}$ is dominated by an integrable function. Since $\left\{f_{n}(x)\right\}_{n \in N(1)}$ was a generic subsequence of $\left\{f_{n}(x)\right\}_{n \in N}$, the proof is complete.

We conclude by giving an example to show that the conditions of the theorem do not require the sequence $\left\{f_{n}(x)\right\}_{n \in N}$ to be convergent or to be dominated by an integrable function. For each $n$ in $N$ let the function $f_{n}(x)$ be defined on the half-open interval $(0,1]$ by 


$$
f_{n}(x)= \begin{cases}0 & \text { if } 0<x<1 /(n+1), \\ n & \text { if } 1 /(n+1) \leqq x<1 / n, \\ (-1)^{n} & \text { if } 1 / n \leqq x \leqq 1\end{cases}
$$

Each $f_{n}(x)$ is integrable with respect to Lebesgue measure on $(0,1]$. It follows that

$$
\begin{gathered}
r(x)=\underset{n \rightarrow \infty}{\lim \sup _{n}} f_{n}(x)=1, \\
s(x)=\underset{n \rightarrow \infty}{\lim \inf _{n}(x)=-1,} \\
R_{n}(x)=\left(r \cup f_{n}\right)(x)= \begin{cases}n \text { if } 1 /(n+1) \leqq x<1 / n, \\
1 \text { otherwise, }\end{cases}
\end{gathered}
$$

and

$$
S_{n}(x)=\left(\operatorname{s\cap } f_{n}\right)(x)=-1 \text {. }
$$

We note that $r(x)$ and $s(x)$ are integrable on $(0,1]$, and that

$$
\lim _{n \rightarrow \infty} \int_{0}^{1} R_{n}(x)=\int_{0}^{1} r(x) .
$$

Let $g(x)$ be a measurable function on $(0,1]$ such that $|g(x)| \equiv 1$. It follows that

$$
\lim _{n \rightarrow \infty} \int_{0}^{1} g(x) R_{n}(x)=\int_{0}^{1} g(x) r(x),
$$

and it is trivial that

$$
\lim _{n \rightarrow \infty} \int_{0}^{1} g(x) S_{n}(x)=\int_{0}^{1} g(x) s(x) .
$$

Let $D_{k}$ be a function on $(0,1]$ which dominates $f_{1}(x), f_{2}(x), \cdots, f_{k}(x)$. Since

$$
\int_{0}^{1} D_{k}(x) \geqq \sum_{n=1}^{k} 1 /(n+1),
$$

it follows that if a function $D(x)$ dominates the sequence $\left\{f_{n}(x)\right\}_{n \in N}$ on $(0,1]$, then $D(x)$ is not integrable.

\section{References}

[1] H. Lebesgue, 'Sur l'intégration des fonctions discontinues', Ann. Ecole Norm. 27 (1910), $361-450$.

[2] B. C. Rennie, 'On dominated convergence', Jour. Australian Math. Soc. 2 (1961-1962), 133 - 136.

[3] S. Saks, Theory of the integral, 2nd ed., Monografje Matematyczne 7, Warsaw, 1937.

Florida State University 